\title{
Cell-cell communication: old mystery and new opportunity
}

\author{
Dongli Song • Dawei Yang • Charles A. Powell • \\ Xiangdong Wang
}

Received: 14 February 2019 / Accepted: 21 February 2019 / Published online: 27 February 2019

(C) Springer Nature B.V. 2019

Cell-cell communication is a critical process that maintains the biological functions and microenvironmental hemostasis of cells, organs, and intact systems. The complexity of cell-cell communication has been investigated for decades and is now accepted as the part of molecular mechanisms of development biology, carcinogenesis, and organ dysfunction The complexity encompasses interactions between heterogeneous cells and between connecting or distant cells, in order to maintain the microenvironmental hemostasis within a special location. For example, telocytes have been suggested as a connecting cell that directly communicate with other types of cells, e.g., epithelia, immune cells, fibroblasts, myocytes, neurocytes, and other organ cells, through secreted mediators, connectors, or ligandreceptor interactions (Wang and Cretoiu 2016). Several recent articles have focused on the role of cell-cell communication in mediating tumor microenvironment complexity, heterogeneity, as well as on tumor recurrence and metastasis (McGranahan and Swanton 2017; EI Rayes et al. 2015). The breakdown of cell-cell communication can compromise retinal homeostasis by

Dongli Song and Dawei Yang contribute to this article equally as the first author

D. Song $\cdot$ D. Yang $\cdot$ C. A. Powell $\cdot$ X. Wang $(\bowtie)$

Zhongshan Hospital Institute for Clinical Science, Shanghai

Medical College, Fudan University, Shanghai, China

e-mail: Xiangdong.wang@clintransmed.org

D. Yang · C. A. Powell

Icahn School of Medicine at Mount Sinai, One Gustave L. Levy

Place, Box 1232, New York, NY 10029, USA changing gene expression of connexin and gap junction intercellular communication, leading to cell apoptosis and breakdown of the blood-retinal barrier (Roy et al. 2017). It is still questioned whether the cell-cell communication can be a new alternative to deeply understand molecular mechanisms of cell-cell interaction, define profiles of intercellular signal networks, and identify cellular function-specific biomarkers and therapeutic targets.

\section{Value of scRNA-seq}

Recent advances in biotechnology provide opportunities to investigate molecular mechanisms of cell-cell communication and discover function-specific target molecules within intercellular networks. For example, function- or character-specific cell-cell communications were recently defined with single-cell measurements. Kumar et al. (2018) used single-cell RNA sequencing (scRNA-seq) to characterize cell-cell communication by analysis of ligand-receptor interactions in the tumor microenvironment of syngeneic mouse models and correlated these observations with tumor growth rates. scRNA-seq as an important approach to define the alterations of transcriptomes, dependent upon the study design, methodology, and scientific questions in the discovery and development of disease-specific dynamic network biomarkers and therapeutic targets (Wang et al. 2017). Notably, scRNA-seq can be used to monitor dynamic phenotypes of single cells per se during evolution, microenvironmental changes, disease progression, 
and therapy, especially with the rapid development of other single-cell technologies (Wang et al. 2017). For example, single-cell pooled CRISPR screening contains the integration of scRNA-seq with CROP-seq, CRISPseq, Perturb-seq, and RISPR/Cas9, to simultaneously measure the perturbation and phenotype of a cell (Wang and Wang 2017).

Kumar et al. (2018) performed an outstanding study to investigate the cell-cell communication between $\mathrm{T}$ cell subsets and their relation to immune infiltration, via the identification and comparison of ligand-receptor interactions, variability across tumors, and relationship to outcome. In a study focused on cardiac cellular interactions, Skelly et al. (2018) utilized scRNA-seq to characterize single-cell transcriptional profiles of the murine non-myocyte cardiac cellular landscape, define diversities of the cardiac cellulome, and extensive networks of intercellular communication.

There are many opportunities to explore the mechanisms of cell-cell communication within the specific organ systems using scRNA-seq to investigate microenvironment alterations caused by inflammation, infection, mechanical injury, or implant. For example, Betsholtz (2018) used scRNA-seq to examine cell-cell signals in blood vessel development and function at a genome-wide and quantitative level of transcriptional diversity. scRNA-seq not only provides single-cell transcriptomes to define cell heterogeneity and new clusters at same cell subtypes and locations, but also identifies profiles of ligand-receptor interaction, cellcell signal networks, and intercellular actions in angiogenesis, carcinogenesis, or tissue repair and regeneration. To address the complexity and diversity of cell populations as well as the difficulty of sample acquisition, Zeng et al. (2018a, b) proposed the artificial intelligent single cell system of computerized databases, digitalized informatics of biological elements, and programmed function and signals of single cells. Smith and Grima (2018) established a mathematical model composed of a stochastic genetic network to investigate cell-cell communications of molecule movements from cell to neighboring cell with a given transport rate or coupling strength. The model demonstrates that single-cell variability is controlled by the strength of cell-cell coupling and is highly dependent upon the variability of the underlying genetic network. It is expected that advances in deep learning approaches and artificial intelligence coupled with increased precision of measurements will enhance the examination of single-cell molecular analysis of intercellular communication. scRNA-seq provides a potential to translate and integrate the intercellular messages, signals, and networks in injured tissues/organs with clinical phenotypes and patient response to therapies.

\section{Potentials of trans-omics}

With the development of single-cell microanalysis techniques, more sensitive, robust, and precise mass spectrometers can be applied to identify putative neuroactive substances, amino acid sequences of a putative neuropeptide, and peptide sequence from single cells that participate in cell-cell communications (Neupert 2018). Neuropeptides from individual single cells in the intra- or inter-cellular interactions and cell-cell communication can be monitored by N-terminal derivatization using 4-sulfophenyl isothiocyanate and subsequent mass spectrometric analysis. Lipid elements dominate compounds in cell secretions and vesicles and thus lipid roles in cell-cell communication should be highly considered. With the development of lipidomics, altered lipidomic profiles have been identified as diseasespecific biomarkers and therapy-associated targets (Lva et al. 2018a, b; Zhang et al. 2018). Several lipidomic pathways involved in the biogenesis and functions of microvesicles and exosomes (Record et al. 2018). Extracellular vesicles are enriched in cholesterol, sphingomyelins, glycosphingolipids, and phosphatidylserine. They contribute to the structure of vesicles, exosome formation, membrane trafficking, and dynamics of release and production within the microenvironment. The lipid composition of exosomes is similar to lipid rafts and has a higher lipid order and higher stability than other extracellular vesicles (Skotland et al. 2019). It is important to classify lipidomic profiles of various extracellular vesicles and to examine the correlation of those profiles with cell phenomes.

With the development of single-cell systems biomedicine (Zeng et al. 2018b; Wang et al. 2018a, b), it is now possible to examine cell-cell communication through the lens of molecular trans-omics that integrates genomics, proteomics, metabolomics, and gene sequencing. Molecular multi-omics describes the networks and correlations among genes, proteins, and lipids at the singlecell level during cell-cell communication. In addition, it is also possible to characterize protein profiles produced from a cell using single-cell proteomics, trace single-cell 
membrane drug delivery, and microenvironmental concentration using porous pen nanodeposition, and to visualize cell-cell interactions using single-cell photoconversion and photoconversions.

Further, molecular trans-omics output can be correlated with clinical trans-omics to provide key insights into disease processes (Wang 2018).

\section{Roles of extracellular vesicles}

Extracellular vesicles play an important role in cell-cell communication in the microenvironment that varies according to the size, membraneous structure, and contents of vesicles, e.g., exosomes $(50-100 \mathrm{~nm})$, microvesicle $(20-1000 \mathrm{~nm})$, membrane particles (50$80 \mathrm{~nm})$, or large membranous vesicles $(\sim 600 \mathrm{~nm})$ or blebbing vesicles (1000-5000 nm). These vesicles contribute to intercellular regulations of metabolisms, signal deliveries, transcriptional gene expressions, and interactions in multi-conditions, including aquatic microbial environments, immune responses, cancer microenvironments, or responses to therapy. Extracellular vesicles carry out autocrine or paracrine cell-to-cell communication within the microenvironment, mainly through proteins, noncoding RNAs (miRNAs), long noncoding RNAs (lncRNAs), mRNAs, DNA, and lipid within vesicles (Bayraktar et al. 2017). For example, miRNAs within extracellular vesicles are produced by cells and transported into neighboring cells with high-density lipoproteins to regulate movement and phenotype. miR-142-3p from lung adenocarcinoma cells was found to be transported to endothelial and fibroblast cells within a tumor to accelerate the process of angiogenesis through inhibition of transforming growth factor receptor 1 and to promote the cancer-associated fibroblast phenotype in lung fibroblast cells independent of TGF $\beta$ signaling (Lawson et al. 2019). The amounts, compositions, characters, and properties of vesicle contents are dependent upon the sources and biological functions of vesicles, although the specificity of vesicles from certain cells is still questioned.

Extracellular vesicles-mediated cell-cell communication impacts physiological changes in health and disease. For example, vesicles secreted from immune cells change adaptive and innate immune responses via exchange of vesicles between different immune cell types in patients with irritable bowel syndrome (Hagiwara et al. 2019). This study indicated that the vesicle contents are relatively specific on basis of cell phenotype and function. Gupta et al. (2019) reported comprehensive proteomic and genomic characterizations of airway epithelial cell secretions and exosomes altered after intercellular exosomal transfer, which were dependent upon the type of airway epithelial cells. Secretions from HTBE or Calu-3 cells were enriched with innate/protective proteins or pathology-associated proteins, while about $20 \%$ of proteins and $4 \%$ miRNAs were altered by HTBE secretions and exosomes after exosome transfer. Future studies will explore whether extracellular vesicles have the specific orientation after the secretion, or whether the specific chemoattractive factors from receptor cells guide the direction of vesicles. Of more importance is to define the specificity of vesicle contents, numbers, and properties to disease types, durations, stages, or phenomes within the context of clinical trials.

\section{Regulation of vesicle production}

The role and function of vesicles in cell-cell communications are more complex than previously understood. Vesicle surface adhesion molecules play an important role in cell-cell recognition, connection, interaction, and transition. For example, actomyosin cytoskeleton tension signaling through adherent junctions can be regulated directly or indirectly by small guanosine triphosphatases (GTPases) of the Rho and Rab families. RhoGTPases-associated cell-cell communication plays an important role in the process of cell migration and regeneration (Combedazou et al. 2017). In particular, the cytoplasmic tyrosine kinase downstream of cellsurface receptors can control intracellular signaling cascades and intercellular communication by regulating the biogenesis and activity of exosomes. The conserved juxtamembrane DEGSY motif of the syndecan cytosolic domain and syntenin tyrosine 46 are directly phosphorylated by the cytoplasmic tyrosine kinase, leading to the activation of ARF6 small GTPase and its effector phospholipase D2 followed by the occurrence of syndecan endocytosis and on syntenin-syndecan endosomal budding (Imjeti et al. 2017). One of the mechanisms by which the exosome is released from an endosomal compartment (MultiVesicular Body) is through the fusion of the compartment plasma membrane regulated by ribonuclease A family member 13 (Ral-1) (Hyenne et al. 2018). Together with SNARE 
protein, ESCRT complex and phospholipase 1D, activated RAL-1, or RalA and RalB contribute to the secretion of exosome-like vesicles.

In conclusion, cell-cell communication is a critical process that controls the signaling and function between cells to maintain microenvironment homeostasis. The intercellular communication is comprised of initiator cells, signal network factors, communicating conditions, and receptor cells. Single-cell RNA sequencing provides single-cell transcriptomes to define cell heterogeneity and new clusters at same cell subtypes and locations, and to identify profiles of ligand-receptor interaction, cell-cell signal networks, and intercellular actions. Molecular multi-/trans-omics may provide new insights and multidimensions of understanding the molecular mechanisms of intercellular communication. Extracellular vesicles as mediators play an important role in cell-cell communication in the microenvironment, according to the size, membranous structure, and contents of vesicles. Although the cellcell communication has been investigated for decades, new technological breakthroughs have yielded new understanding of its mechanisms that provide opportunities for the identification and development of disease-specific biomarkers and therapeutic targets.

Publisher's note Springer Nature remains neutral with regard to jurisdictional claims in published maps and institutional affiliations.

\section{References}

Bayraktar R, Van Roosbroeck K, Calin GA. Cell-to-cell communication: microRNAs as hormones. Mol Oncol. 2017;11(12): 1673-86. https://doi.org/10.1002/1878-0261.12144.

Betsholtz C. Cell-cell signaling in blood vessel development and function. EMBO Mol Med. 2018;10(3):e8610. https://doi. org/10.15252/emmm.201708610.

Combedazou A, Gayral S, Colombié N, Fougerat A, Laffargue M, Ramel D. Small GTPases orchestrate cell-cell communication during collective cell movement. Small GTPases. $2017 ; 17: 1-10$. https://doi org/10.1080 /21541248.2017.1366965.

EI Rayes T, Catena R, Lee S, Stawowczyk M, Joshi N, Fischbach $\mathrm{C}$, et al. Lung inflammation promotes metastasis through neutrophil protease-mediated degradation of Tsp-1. Proc Natl Acad Sci U S A. 2015;112(52):16000-5. https://doi. org/10.1073/pnas.1507294112.

Gupta R, Radicioni G, Abdelwahab S, Dang H, Carpenter J, Chua $\mathrm{M}$, et al. Intercellular communication between airway epithelial Cells is mediated by exosome-like vesicles. Am J Respir Cell Mol Biol. 2019 Feb;60(2):209-20. https://doi. org/10.1165/rcmb.2018-0156OC.
Hagiwara SI, Hasdemir B, Heyman MB, Chang L, Bhargava A. Plasma Corticotropin-releasing factor receptors and B7-2 $2^{+}$ extracellular vesicles in blood correlate with irritable bowel syndrome disease severity. Cells. 2019;8(2):E101. https://doi.org/10.3390/cells8020101.

Hyenne V, Labouesse M, Goetz JG. The small GTPase Ral orchestrates MVB biogenesis and exosome secretion. Small GTPases. 2018;9(6):445-51. https://doi.org/10.1080 /21541248.2016.1251378.

Imjeti NS, Menck K, Egea-Jimenez AL, Lecointre C, Lembo F, Bouguenina H, et al. Syntenin mediates SRC function in exosomal cell-to-cell communication. Proc Natl Acad Sci U S A. 2017;114(47):12495-500. https://doi.org/10.1073 /pnas. 1713433114.

Kumar MP, Du J, Lagoudas G, Jiao Y, Sawyer A, Drummond DC, et al. Analysis of single-cell RNA-Seq identifies cell-cell communication associated with tumor characteristics. Cell Rep. 2018;25(6):1458-1468.e4. https://doi.org/10.1016/j. celrep.2018.10.047.

Lawson J, Dickman C, Towle R, Jabalee J, Javer A, Garnis C. Extracellular vesicle secretion of miR-142-3p from lung adenocarcinoma cells induces tumor promoting changes in the stroma through cell-cell communication. Mol Carcinog. 2019;58(3):376-87. https://doi.org/10.1002/mc.22935.

Lv J, Gao D, Zhang Y, Wu D, Shen L, Wang X. Heterogeneity of lipidomic profiles among lung cancer subtypes of patients. J Cell Mol Med. 2018a;22(10):5155-9. https://doi.org/10.1111 /jcmm.13782.

Lv J, Zhang L, Yan F, Wang X. Clinical lipidomics: a new way to diagnose human diseases. Clin Transl Med. 2018b;7(1):12. https://doi.org/10.1186/s40169-018-0190-9.

McGranahan N, Swanton C. Clonal heterogeneity and tumor evolution: past, present, and the future. Cell. 2017;168:61328.

Neupert S. Single cell Peptidomics: approach for peptide identification by N-terminal peptide derivatization. Methods Mol Biol. 2018;1719:369-78. https://doi.org/10.1007/978-14939-7537-2_25.

Record M, Silvente-Poirot S, Poirot M, Wakelam MJO. Extracellular vesicles: lipids as key components of their biogenesis and functions. J Lipid Res. 2018;59(8):1316-24. https://doi.org/10.1194/jlr.E086173.

Roy S, Kim D, Lim R. Cell-cell communication in diabetic retinopathy. Vis Res. 2017;139:115-22. https://doi.org/10.1016 j.visres.2017.04.014.

Skelly DA, Squiers GT, McLellan MA, Bolisetty MT, Robson P, Rosenthal NA, et al. Single-cell transcriptional profiling reveals cellular diversity and intercommunication in the mouse heart. Cell Rep. 2018;22(3):600-10. https://doi.org/10.1016 j.celrep.2017.12.072.

Skotland T, Hessvik NP, Sandvig K, Llorente A. Exosomal lipid composition and the role of ether lipids and phosphoinositides in exosome biology. J Lipid Res. 2019;60(1):9-18. https://doi.org/10.1194/jlr.R084343.

Smith S, Grima R. Single-cell variability in multicellular organisms. Nat Commun. 2018;9(1):345. https://doi.org/10.1038 /s41467-017-02710-x.

Wang X. Clinical trans-omics: an integration of clinical phenomes with molecular multiomics. Cell Biol Toxicol. 2018 Jun;34(3): 163-6. https://doi.org/10.1007/s10565-018-9431-3. 
Wang X, Cretoiu D. Telocytes: connecting cells, edited series title: advances in experimental medicine and biology. 2016; V913. eBook ISBN978-981-10-1061-3, Hardcover ISBN: 978981-10-1060-6, Softcover ISBN: 978-981-10-9318-0, Series ISSN: 0065-2598. Singapore: Springer. https://doi. org/10.1007/978-981-10-1061-3.

Wang W, Wang X. Single-cell CRISPR screening in drug resistance. Cell Biol Toxicol. 2017;33(3):207-10. https://doi. org/10.1007/s10565-017-9396-7.

Wang W, Zhu B, Wang X. Dynamic phenotypes: illustrating a single-cell odyssey. Cell Biol Toxicol. 2017;33(5):423-7. https://doi.org/10.1007/s10565-017-9400-2.

Wang Y, Li L, Wang X, Gu J. Can the single cell make biomedicine different? Adv Exp Med Biol. 2018a;1068:1-6. https://doi.org/10.1007/978-981-13-0502-3_1.
Wang W, Gao D, Wang X. Can single-cell RNA sequencing crack the mystery of cells? Cell Biol Toxicol. 2018b;34(1):1-6. https://doi.org/10.1007/s10565-017-9404-y.

Zeng Y, Chen X, Gao H, Wang X. An artificial intelligent single cell is part of the cell dream world. Cell Biol Toxicol. 2018a;34(4):247-9. https://doi.org/10.1007/s10565-0189433-1.

Zeng Y, Chen X, Wang X. Roles of single cell systems biomedicine in lung diseases. Adv Exp Med Biol. 2018b;1068:17785. https://doi.org/10.1007/978-981-13-0502-3_15.

Zhang L, Han X, Wang X. Is the clinical lipidomics a potential goldmine? Cell Biol Toxicol. 2018 Dec;34(6):421-3. https://doi.org/10.1007/s10565-018-9441-1. 